\title{
The downstream effects of COVID-19: a call for supporting family wellbeing in the NICU
}

\author{
Carmina Erdei $\mathbb{C}^{1,2} \cdot$ Cindy H. Liu ${ }^{1,2}$
}

Received: 1 May 2020 / Revised: 23 June 2020 / Accepted: 17 July 2020 / Published online: 24 July 2020

(c) Springer Nature America, Inc. 2020

Parents of NICU infants are a vulnerable population from a psychological perspective, and often experience high levels of acute stress, depression, anxiety, and post-traumatic stress. The added burden of the current SARS CoV-2 disease (COVID19) pandemic is likely to exacerbate these issues, with potential implications for the wellbeing of infants and families in the short- and long-term. In this paper, we propose utilizing the stress contagion framework and consider how psychosocial stress can "spill over" into the parent-infant relationship domain, which can impact child development and family wellbeing longer term. As the effects of the pandemic will likely persist well beyond the acute stage, we offer advocacy points and general guidelines for healthcare professionals to consider in their quest to mitigate stress and build resilience in NICU families.

\section{Introduction}

The novel coronavirus SARS-CoV-2 disease (COVID-19) pandemic has had a global impact resulting in stress on health systems worldwide. Recent data from China, the first country to experience a large-scale outbreak, suggested that newborns remain susceptible to SARS-CoV-2, although symptoms are milder and outcomes less severe compared to adults [1]. Transmission is thought to occur primarily horizontally by caregiver-to-infant postnatal transmission via respiratory droplets $[1,2]$.

Although newborns may be less impacted by COVID-19 directly, there is concern that the pandemic and the necessary public health measures employed in response may negatively impact the psychosocial health of high-risk infants and their families in the Neonatal Intensive Care Unit (NICU). The impact of early adversity on infant brain development is well recognized [3], and recommendations are in place to mitigate these effects through NICU familycentered developmental care models $[4,5,6]$.

Parents are critical to the health and wellbeing of their infants. From a psychological perspective, families of NICU infants are a vulnerable population, as they experience

\footnotetext{
Carmina Erdei

CErdei@bwh.harvard.edu

Brigham and Women's Hospital, Boston, MA, USA

2 Harvard Medical School, Boston, MA, USA
}

trauma due to separation from their infants alongside the stress regarding their medical conditions and related interventions [7]. Many NICU families experience altered perinatal parent mental health in the form of acute stress, anxiety, depression, and/or post-traumatic stress [7, 8]. This is associated with an elevated risk for subsequent impaired child development and poorer family functioning [8, 9], which led to a recent call for enhanced mental health support for NICU families $[4,10]$. In this context, the current COVID-19 pandemic acts as a sudden, new-onset major environmental stressor superimposed on preexisting high levels of NICU family psychological distress.

A crucial public health measure in the fight against COVID-19 is the social distancing imperative. This has resulted in difficulties with transportation and access to hospitals in some communities. Within some units, it has also led to more limited family access to the NICU, challenging family-centered developmental care practices. This, in turn, may affect the ability for families to cope with pandemic-related stress, thus compounding the stress of having an infant in the NICU, and further impeding their confidence as primary caregivers. The parental experience of navigating the changes in the healthcare system, combined with potential new financial and other constraints during the COVID-19 pandemic can spill over to how they engage and connect with their infant, which is referred to as stress contagion [11, 12].

Against this background, several issues can impact parental wellbeing in the NICU. We highlight two major sources of stress: 
1. External factors such as public health measures and institutional policies that directly or indirectly impact NICU parents. Examples include (a) limited presence and availability of families in the NICU due to caregiver SARS-CoV-2 positive status and/or COVID-19 illness, (b) voluntary separation of SARS-CoV-2 positive caregivers from their infants to minimize viral transmission, (c) tightened transportation and hospital visitation policies to reduce community transmission, and (d) more limited extended family and community support.

2. Internal factors including individual characteristics that lead to parents experiencing different levels of stress, different sources of stress, and variable ability to adjust to the "new normal". Families often vary in their ability to employ coping strategies to reframe stress and hone their capacity to engage and connect with their infant in the face of adversity.

When the interplay between these factors exceeds a family's capacity to manage stress, this may spill over to affect the complex relationship-building process that occurs after every birth, and is especially vulnerable for disruption in the NICU. Nurturing experiences including breastfeeding, kangaroo mother's care, or parents reading/ talking with their infant which are routinely employed to mitigate stress may occur less frequently. Parents might experience heightened anxiety and extreme worry about their infant's health in the pandemic era that is even higher than that typically experienced by parents in the NICU, an aspect that deserves further investigation. Given the negative downstream effects that NICU stress contagion may have on child development and family functioning, professionals should consider advocacy and further action in their quest to foster family resilience in the COVID-19 era.

\section{Key guidelines}

(1) Practice shared decision-making for every aspect of care.

Of particular note, this should include the psychosocial domain. Healthcare providers should assess sources of stress and perceived risks from NICU parents, and partner with families to devise individualized care plans through shared decision-making. Systematic assessment of NICU families psychosocial stress, and enhanced collaboration with mental health specialists will aid this goal.

We note that disadvantaged families may disproportionately experience stress contagion, and additional supportive mechanisms may be necessary to meet each family's needs. Issues unique to or exacerbated by the COVID-19 pandemic include:

- Increased transmission of SARS-CoV-2 in family's community.

- Limited access to technology for virtual communication and/or care.

- Limited access to support systems in family's community.

- Exacerbation of preexisting mental health issues.

- Communication challenges for non-English speaking families.

- Poverty, housing, or food insecurity.

- More limited ability to be present in the NICU if dependent on public transportation due to loss of income and/or travel restrictions.

(2) Increase support to mitigate stress contagion.

If infrastructure and model of care permits, NICUs can temporarily consider increasing mental health support in the NICU during this stressful period, to facilitate development of family coping strategies and resilience.

- Emphasize basic wellness strategies: encourage parent wellbeing, healthy diet, sleep, exercise, virtual social connectedness, mindfulness, and self-compassion.

- Increase virtual and/or on-site social work and other mental health and psychosocial support, including behavioral health and virtual peer-topeer connection.

- Capitalize on the critical role that fathers/partners play both in the infants' care and support of mothers.

- Consider assessment of baseline NICU families mental health, as well as identification of pandemicrelated superimposed stressors unique to each family; evaluate which superimposed stressors offer opportunities for intervention.

- Optimize family-centered developmental care opportunities, support relationship-building within the family as much as safely possible, and prioritize interventions that meet the needs of the entire family unit. Consider positive multisensory interventions facilitated by staff and technology where appropriate, when family presence is limited.

(3) Prioritize stress mitigation for healthcare professionals, so they can themselves remain resilient and provide emotional support for NICU families [13]. 


\section{Summary}

As the neonatal community has expeditiously employed novel strategies to meet the medical needs of infants affected by COVID-19, it is important to also consider the psychological short- and long-term effects of the pandemic on NICU families. The mental health aftermath of the pandemic will likely persist long after the acute phase, and it may disproportionately affect the vulnerable NICU population. As stress contagion in the NICU can negatively impact parent-infant relationships, strategies are needed to mitigate it in order to optimize child development and family resilience during this unparalleled period. Lastly, innovation and discovery can often arise during crises. As the acute phase of the pandemic concludes, professionals might consider which COVID-19 era interventions may prove beneficial in enhancing current family-centered developmental care practices in the NICU and are worth developing further.

Acknowledgements We would like to thank Dr. Terrie Inder, MBChB, MD, Chair of Department of Pediatric Newborn Medicine at Brigham and Women's Hospital and Mary Ellen Avery Professor of Pediatrics at Harvard Medical School, for her valuable consultation and feedback in the preparation of this paper. We are also deeply appreciative to the Nunnally family and Weinberg family for their generous support of the Brigham and Women's Hospital NICU programs.

\section{Compliance with ethical standards}

Conflict of interest The authors declare that they have no conflict of interest.

Publisher's note Springer Nature remains neutral with regard to jurisdictional claims in published maps and institutional affiliations.

\section{References}

1. Zhang ZJ, Yu XJ, Fu T, Liu Y, Jiang Y, Yang BX, et al. Novel coronavirus infection in newborn babies under 28 days in China. Eur Respir J. 2020;55:2000697.

2. Mimouni F, Lakshminrusimha S, Pearlman SA, Raju T, Gallagher PG, Mendlovic J. Perinatal aspects on the covid-19 pandemic: a practical resource for perinatal-neonatal specialists. J Perinatol. 2020;40:820-6.

3. Tierney AL, Nelson CA III. Brain development and the role of experience in the early years. Zero Three. 2009;30:9.

4. Hall SL, Hynan MT, Phillips R, Lassen S, Craig JW, Goyer E, et al. The neonatal intensive parenting unit: an introduction. J Perinatol. 2017;37:1259-64.

5. Davidson JE, Aslakson RA, Long AC, Puntillo KA, Kross EK, Hart J, et al. Guidelines for family-centered care in the neonatal, pediatric, and adult ICU. Crit Care Med. 2017;45:103-28.

6. Als H, B McAnulty G. The newborn individualized developmental care and assessment program (NIDCAP) with kangaroo mother care (KMC): comprehensive care for preterm infants. Curr Womens Health Rev. 2011;7:288-301.

7. Rogers $\mathrm{CE}$, Kidokoro $\mathrm{H}$, Wallendorf $\mathrm{M}$, Inder TE. Identifying mothers of very preterm infants at-risk for postpartum depression and anxiety before discharge. J Perinatol. 2013;33:171-6.

8. Treyvaud K, Lee KJ, Doyle LW, Anderson PJ. Very preterm birth influences parental mental health and family outcomes seven years after birth. J Pediatrics. 2014;164:515-21.

9. McManus BM, Poehlmann J. Parent-child interaction, maternal depressive symptoms and preterm infant cognitive function. Infant Behav Dev. 2012;35:489-98.

10. Treyvaud K, Spittle A, Anderson PJ, O'Brien K. A multilayered approach is needed in the NICU to support parents after the preterm birth of their infant. Early Hum Dev. 2019;139:104838.

11. Bolger N, DeLongis A, Kessler RC, Wethington E. The contagion of stress across multiple roles. J Marriage Fam. 1989;51:175-83.

12. Liu CH, Doan SN. Psychosocial stress contagion in children and families during the COVID-19 pandemic. Clin Pediatrics. 2020; 59:853-5.

13. Canady VA. Amid COVID-19, self-care as important for providers as it is for patients. Ment Health Wkly. 2020;30:1-4. 